\title{
The Role of On-line Urban Signal Control in Congestion and Incident Detection and Management
}

\author{
Authors: Christiane Bielefeldt, The MVA Consultancy, Edinburgh \\ Dave Bretherton, Transport Research Laboratory, Crowthorne \\ Colin Toomey, Traffic Control Systems Unit, London
}

\section{The Problem}

Urban congestion is one of today's main traffic problems. Congestion affects in particular radial routes to the town centres in the morning and afternoon peak, and many central business districts even outside the peak periods. The main cause for congestion is oversaturation of the networks, but the situation is worsened when incidents occur.

The solutions that are potentially the most effective ones are either increasing the road supply or decreasing the traffic demand. The first is a solution of the past, and, today, generally no longer an option in urban areas because of both physical and environmental constraints, while decreasing traffic demand throigh demand management is certainly an option for the future. Possibilities for restraining car traffic in urban areas are much discussed today: either through reducing the network capacity for private cars, eg through traffic calming or the introduction of bus lanes, or through pricing policies, either for car parking or as some form of road pricing. The first of these measures are already being implemented in many places today, but were nowhere sufficient to substantially alleviate congestion problems, and in some places may have even aggrevated them through increased pressure on the remaining road space. And comprehensive urban road pricing that could have a major impact on congestion reduction will not be widely introduced for a long time.

The implication of all this is that Urban Traffic Control (UTC) will have to cope with ever increasing traffic problems for years to come. UTC comprises, of course, a number of elements, and parking guidance or rerouteing will help in many cases to prevent or reduce congestion. However, network signal control is still at the very core of any UTC system, and will have to able to deal with congestion.

Most of the congested routes and road networks in urban areas are equipped with traffic signals. However, the signal programs are generally not well prepared to deal adequately with congestion and, even more so, with incident problems.

Traditional signal control on the basis of signal plans that have been prepared off-line and are selected on-line on a time-dependent or maybe traffic-dependent basis can, to a certain degree, be adequate for responding to recurrent congestion, which follows predictable patterns. In cases of incidents, however, the number of possibilites through different incident locations, types and severities and the particular traffic situations, in which these incidents occur, is nearly unlimited, and it is impossible to provide sets of signal plans that respond adequately to this variety of incident situations in advance. It is in these cases that on-line signal control, with all its flexibility, shows some of its biggest advantages.

All of the current main contenders for on-line signal network control in Europe (SCOOT, SCATS, UTOPIA, PRODYN and MOTION) have originally been designed to optimise 
unsaturated traffic. They are doing this in different ways and using different types of optimisation criteria, and some of them have been adapted in the meantime to enable them to react to some extent to congestion and incidents. However, none of them, so far, contains general procedures for Congestion and Incident Management (CIM).

\section{The COSMOS Project}

COSMOS (Congestion Management Strategies and Methods in Urban Sites) is one of the European Commission's ATT projects in the 4th Framework Programme of Research. The COSMOS project will develop, validate and demonstrate new procedures for reducing and, where possible, preventing congestion caused by incidents and oversaturation in densely trafficked urban areas. These procedures comprise:

- $\quad$ enhanced algorithms for Automatic Incident and Congestion Detection (ACID),

- special modules for Congestion and Incident Management (CIM) which can be included in any on-line network signal control systems, and

- $\quad$ strategies for rerouteing traffic to make the best use out of the capacity at junctions and in the links between them.

The project started with the definition of the user requirements for congestion and incident management. Based on these requirements, the strategic interfaces between urban signal control and other UTC systems (in particular rerouteing either with VMS or through invehicle route guidance, but also with public transport and parking management) in the specific context of CIM were to be defined. This integration concept comprises both data flows and the requirements for prediction and modelling tools and strategies for the development of control decisions.

In parallel, requirements and possibilities for enhancing the existing ACID capabilities in SCOOT, UTOPIA and MOTION were defined. The INGRID automatic incident detection and ASTRID traffic monitoring system used in SCOOT are described and discussed in another paper in this colloquium. In UTOPIA, enhancements will be made to the observer modules at both local and central level, including the information flow needed between them. In MOTION, the main improvement envisaged is to refine the definition of both congestion and incidents with regard to their spread and severity. In all three systems, particular emphasis is placed on the recognition of area wide effects of incidents and congestion.

In the next step, the general principles for CIM strategies were to be elaborated. These principles take account of both the possibilities of urban signal control and the restrictions set by the ACID systems and by the demands of other UTC systems. Based on these general principles, a set of detailed control strategies for specific congestion and incident scenarios is now under development, and will be described further in this paper. This common development will branch out into the specification of algorithms and the building of three separate demonstrators for SCOOT, MOTION and UTOPIA. In parallel, rerouteing strategies will be prepared for the implementation in the test sites. 
Finally, prototypes of these systems and strategies will be installed in London, Piraeus and Torino for verification and demonstration. The subsequent evaluation will establish the performance of the new systems and varied traffic conditions.

\section{The Possibilities for Signal Control}

All of the three systems SCOOT, UTOPIA and MOTION have very different philosophies, very different methods of optimising signal times, and are using different technologies. It was decided early in the conception of the COSMOS project that these existing systems should not be overridden and that any future development work should be sympathetic to each system's rationale. Therefore, for all future work in COSMOS, every system should have, wherever appropriate, the same common CIM strategies, the so-called Common Control Strategies (CCS), but the realisation of these strategies must be carried out under the normal rules of the individual system.

To reflect these differences, four levels of operation were defined: strategies, tactics, tools, and realisation.

\section{The strategies}

The strategies for CIM form the top level of this hierarchy, and have to reflect the strategic requirements of the system operators. They define the rules

\footnotetext{
- under which circumstances

- which tactics and

- which tools will be used

- in which sequence and

- in which combination.
}

In very general terms, the strategies fall into the three categories congestion avoidance, congestion reduction, and congestion shift. Congestion avoidance can comprise both: strategies based on a traffic prediction of the imminent onset of congestion, and strategies that avoid a spread of congestion from the primary link into the wider network. Congestion reduction involves proactive control measures which will change network signal timings to clear out queues from sensitive areas. Congestion shift is based on the premise that whereas congestion in one place may spread rapidly out of control, there may be other places where its negative impact can be more easily tolerated and/or where its knock-on effects, particularly on public transport, are minimised. Typically this will involve the use of queue storage in high capacity long links or even outside the controlled area. Measures to implement this strategy would include rerouteing using remote signing.

The optimal utilisation of all strategies depends on high quality congestion monitoring, and incident detection and location. Congestion avoidance requires, furthermore, depends on the availability of elements of congestion prediction in the UTC system. 
To define the circumstances under which certain tactics and tools can be deployed, a number of congestion and incident scenarios have been defined. Fig 1 shows the latest draft of a so-called 'scenario decision tree'. This decision tree guides through a number of questions that define the features of the different scenarios: scenarios $\mathrm{C} 1$ to $\mathrm{C} 5$ for links with primary congestion, I1 to $\mathrm{I10}$ for links with incidents, and S1 to S5 for links that suffer secondary or blocking-back effects from primary congestion or incidents further downstream.

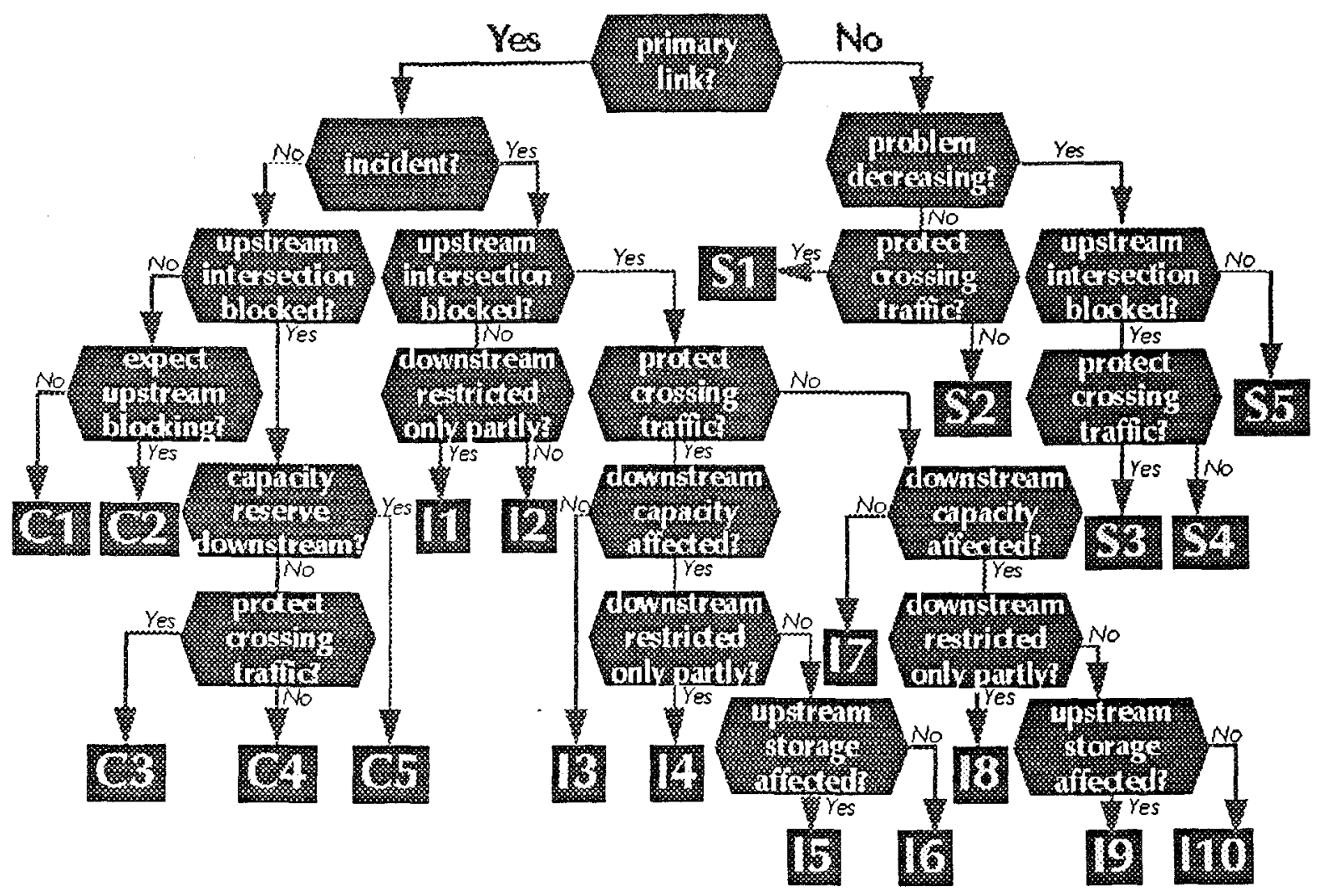

Figure 1: Scenario Decision Tree (Draft)

For each of the scenarios, a number of 'responses' are available, with each response consisting of one tactic and anything between one and four tools that the particular tactic can use in these circumstances. It should be noted, however, that the responses do not try to solve the problems attached to a particular scenario in a holistic approach, but, instead, refer to individual specific features of each scenario. Morever, many features will be common to a number of scenarios. In so far, the tree is more a device to show which traffic situations can be addressed than a blueprint for designing the structure of the algorithms and the software.

The scenario tree and the responses are, at the time of this colloquium (June 1997), still undergoing the last revision of their detailed specification. 


\section{The tactics}

The tactics are the means that can be employed by the strategies to achieve particular goals in particular situations. They are mainly applicable to one intersection, or in some cases to a group of intersections which form a subset of the overall network:

- Entry gating is designed to prevent traffic from entering a network so as to avoid oversaturation within it, or if implemented at a later point, at least to limit congestion in the network. Gating can be implemented just at the local intersection, progressively along an arterial, or at the edges of the controlled area to protect a more distant part of the network.

- Opening an exit will allow traffic to leave a congested network more rapidly than with the standard signalisation. Like gating, it can be applied either as a local tactic, or a progressive linear one.

- Preventing blocking crossing traffic is meant to start the red to the main road feeding the congested link before the exit to the junction becomes blocked, so that the traffic trying to cross the congested stream can do so unhindered by fully utilising its green time.

- Giving priority to a particular traffic stream is designed to provide green time for traffic that tries to join the congested stream from a specific upstream link when they can effectively use this green time, ie when the end of the downstream queue is moving.

- Ensuring full utilisation of exit capacity is a tactic that applies to short links where congestion is building up because the existing green time cannot be fully used due to an unbalance between green time and storage capacity.

\section{The tools}

The tools that are used by the tactics relate to the elements of the signal plan and the ways in which they can be modified. The twelve tools that are available are:

- Double cycling, which can be used when traffic flow shall be split into many small platoons either because of the lack of storage space for long queues or to condense the platoons crossing the stopline.

- Decreasing the cycle time for the whole network, or a subnetwork where this can be created with reasonable borders, may be an alternative when double cycling is not feasible for some reason eg because it would reduce the capacity too much due to large intergreen times.

- Increasing the cycle time is the standard response when traffic volumes increase in a network.

- Doubling the cycle time can create additional capacity beyond the normal limits that may be useful on long exit links of congested networks.

- The purpose of a reduced offset is to avoid congestion through avoiding blocking back together with unused green time at the downstream intersection that leads to inefficient use of the green time upstream and, if demand is high, to congestion building up upstream.

- The balanced offset can in some cases be designed to reduce congestion, in others it may only help to shift it to those links where it does less harm, ie generally from 
short to long links or from links with residential use to those with mixed or industrial use.

- Another possibility to use the offset is for platoon compression to protect short links, if the green time upstream is larger than downstream

- Double green can be used for the same purposes as double cycling (To 1) as an alternative where three or more separate stages per cycle are needed.

- $\quad$ Furthermore, the phase sequence might be modified to support the creation of a particular offset, if this helps to reduce stops and delays for the opposite direction.

- Increasing green time at the downstream signal, within the given cycle time either in line or overproportionately with increasing the cycle time, is a standard tool to increase capacity at the downstream signal in a congested link

- Decreasing the green time at the upstream signals can be useful to avoid oversaturation for the downstream link with the consequence of blocking back, and in those cases for which double cycling, platoon compression or double green have to be considered.

- Adjustment of the green split according to queuing capacity may be useful in particular when one or more links at an intersection are very short.

\section{The realisation}

Finally, because of the previously mentioned differences in the methodological approaches and technologies between SCOOT, UTOPIA and MOTION, the common control strategies and tactical decisions will be realised through the different techniques used by those control systems.

\section{Outlook}

The definition of the scenarios, for which the Common Control Strategies can be utilised, will be finalised in the next weeks. Discussions about the applicability of scenarios and strategies in the three test sites are currently underway. A public COSMOS deliverable which will describe the scenarios, the strategies, the tactics and the tools in detail will be published in October 1997.

Those strategies that can be implemented in the test sites will be integrated in SCOOT, UTOPIA and MOTION during the next year. The verification phase will start in mid 1998, and the final results of the COSMOS project, including an evaluation of the benefits of the new Congestion and Incident Management modules, will be available in April 1999. 


\section{The realisation}

Finally, because of the previously mentioned differences in the methodological approaches and technologies between SCOOT, UTOPIA and MOTION, the common control strategies and tactical decisions will be realised through the different techniques used by those control systems.

\section{Outlook}

The definition of the scenarios, for which the Common Control Strategies can be utilised, will be finalised in the next weeks. Discussions about the applicability of scenarios and strategies in the three test sites are currently underway. A public COSMOS deliverable which will describe the scenarios, the strategies, the tactics and the tools in detail will be published in October 1997.

Those strategies that can be implemented in the test sites will be integrated in SCOOT, UTOPIA and MOTION during the next year. The verification phase will start in mid 1998, and the final results of the COSMOS project, including an evaluation of the benefits of the new Congestion and Incident Management modules, will be available in April 1999. 